\title{
Bioética narrativa y salud intercultural mapuche Aportes a su fundamentación y complementación
}

\author{
ÁlVARO RAMIS OLIVOS \\ Universidad de Santiago de Chile (Chile) \\ alvaro.ramis@usach.cl
}

\section{Resumen}

Este artículo tiene como objetivo sistematizar los avances del enfoque narrativo en bioética con el fin de proponer una fundamentación de las prácticas de salud intercultural que facilite su complementariedad con el conjunto del sistema sanitario. Para ello revisará críticamente los fundamentos de la bioética narrativa y su vinculación con las corrientes hermenéuticas en las éticas aplicadas y con el movimiento de la medicina narrativa. A la vez relacionará el enfoque narrativo con el paradigma intercultural en salud, en orden a evaluar sus capacidades de complementación y articulación con la sabiduría ancestral mapuche, desde su raigambre y especificidad. Finalmente informará sobre las posibilidades de desarrollo de una bioética narrativa en clave intercultural, adecuada a las prácticas en el ámbito sanitario que se basan en el acervo de la sabiduría ancestral mapuche.

Palabras clave: Bioética narrativa, Hermenéutica, Salud intercultural, éticas aplicadas.

\section{Narrative bioethics and Mapuche intercultural bealth contributions to the groundwork and complementation}

\begin{abstract}
This article aims to systematize the progress of the narrative approach in bioethics in order to propose a groundwork for intercultural health practices. Seeks propose a basis for intercultural health practices that facilitate the complementarity of the health system through the recovery of ancestral Mapuche Worldview, their roots and specificity. It will critically review the basics of narrative bioetbics and its relationship with the hermeneutical currents in applied ethics and the movement of Narrative Medicine. At the same time relates the narrative approach to the intercultural paradigm in health, in order to assess its capabilities complementation and articulation. And finally it will report on the possibilities of developing an intercultural key Narrative Bioethics appropriate to the intercultural practices in health care based on the acquis of the Mapuche Ancestral Wisdom.
\end{abstract}

Key Words: Narrative Bioethics, Hermeneutics, Intercultural Health, Applied Ethics.

Doctor en Filosofía por la Universidad de Valencia (España). Profesor en el programa de bachillerato de la Universidad de Santiago y en la Universidad Alberto Hurtado. Artículos recientes: "Lo que sin ser de nadie es de todos. Una interpretación de la idea de «bienes comunes de la humanidad" (2014) y "Los bienes comunes intangibles en el capitalismo cognitivo" (2014).

Recibido: 2/Diciembre/2015 - Aceptado: 8/Enero/2016 


\section{Introducción}

Por bioética narrativa se entiende una perspectiva teórico-práctica innovadora, que integra críticamente la medicina narrativa y las corrientes hermenéuticas y discursivas en las éticas aplicadas, con el fin de complementar al enfoque principialista, que ya tiene un largo desarrollo en bioética. A su vez, la medicina narrativa es un movimiento académico que fomenta la sensibilización ante las percepciones del paciente y promueve la adquisición de "competencias narrativas", que atiendan a las vivencias histórico-biográficas ligadas a las experiencias de salud y enfermedad.

La hipótesis basal de este enfoque bioético radica en que la narratividad brinda criterios hermenéuticos que amplían el campo deliberativo de las éticas de la vida, aquilatando las nociones de salud, enfermedad, bienestar y buen vivir. De esa manera se revalorizan las dimensiones emocionales, relacionales y cosmovisionales, ensanchando los enfoques interpretativos, sin abandonar el rigor conceptual y metodológico.

La bioética narrativa constituye un campo de estudios, reflexión y propuestas interpretativas que ha ganado visibilidad en los últimos años al calor de los debates referidos a la calidad de la atención médica, la integración de una pluralidad de modelos de intervención clínica y ante la necesidad de reconocer al paciente como protagonista de todo proceso terapéutico.

Se trata de un modelo interpretativo que retoma aspectos claves de lo más aquilatado de la tradición hipocrática, que concibe la salud humana como eyctasía, un proceso de armonía en la naturaleza humana, en tanto equilibrio en los humores corporales internos, pero también un estado de armonía con el entorno intersubjetivo y relacional. De igual forma Hipócrates entiende la enfermedad (nósas) como un proceso que se despliega en la gradualidad del tiempo. Se trata de cambios que poseen causas que exigen ser descifradas en sus aspectos típicos y específicos. Y es el propio sujeto de esos cambios quién puede aportar las claves del círculo hermenéutico de la salud y la enfermedad.

De allí que tras las exploraciones de la bioética narrativa se hagan eco de antiguas reflexiones médicas que hoy asumen renovado valor. Vale recordar que alguna ocasión el doctor Gregorio Marañón, consultado respecto a la innovación médica más importante de los últimos años respondió lacónicamente «la silla» ya que a su juicio esa es la herramienta que permite sentarse al lado del paciente, escucharlo y explorarlo (Pérez Oliva: 1989).

Gracias a estas aportaciones el paradigma narrativo en bioética asume que los eventos vitales cobran pleno sentido dentro de historias 
complejas, y el reconociendo de esos relatos da acceso a la identidad biográfica de los sujetos, permitiendo interpretar y deliberar sobre los valores de las personas y sus mundos vitales. Por esta razón, esta perspectiva puede contribuir a la integración de los sistemas de salud de pueblos y culturas con diferentes intereses y tradiciones valorativas, especialmente en contextos culturales asimétricos. Ello presupone que el reconocimiento intercultural no sólo se funda en una necesidad terapéutica, sino ante todo en una exigencia de justicia, por lo cual las prácticas interculturales en salud no se deben conceptualizar como procesos excepcionales o mero recursos episódicos o incidentales.

\section{Situación de la bioética narrativa}

En las sociedades tradicionales las relaciones médico-paciente se comprendían desde una ética del cuidado que vista desde la actualidad se tiende a interpretar como paternalista. En efecto, el Corpum Hipocráticum describe el rol del médico en su diálogo anamnésico desde una actitud de desconfianza ante el paciente:

Haz todo en calma y orden ocultando al enfermo durante tu actuación la mayoría de las cosas. Dale las órdenes oportunas con amabilidad y dulzura y distrae su atención; repréndele a veces estricta y severa mente pero otras anímale con solicitud y habilidad sin mostrarle nada de lo que va a pasar ni de su estado actual pues muchos acuden a otros médicos por causa de esa declaración antes mencionada, del pronóstico de su presente y su futuro. (Hipócrates, 2003:9)

Frente a esta doctrina la medicina moderna reaccionó introduciendo de forma progresiva el principio de autonomía del paciente, fundado en una concepción de la autonomía moral que presupone la capacidad universal de servirse del propio entendimiento. La judicialización de estos principios se plasmó en el "Informe Belmont" (1978) propuesto por la Comisión Nacional para la Protección de los Sujetos Humanos de Investigación Biomédica y del Comportamiento, del Congreso de Estados Unidos. Este documento distinguió tres principios básicos: respeto por las personas, beneficencia y justicia. Posteriormente, profundizando en esta propuesta Beauchamp y Childress propusieron en 1979 cuatro principios aplicables a la práctica asistencial: no maleficencia, beneficencia, autonomía y justicia también llamados "principios de Georgetown" (Beauchamp \& Childress: 1994). Como es de conocimiento, en la actualidad los dilemas éticos que presentan las prácticas biomédicas son examinados bajo un marco común internacional, basado en estos principios. 
A pesar de su amplio reconocimiento y utilización, este enfoque ha sido criticado desde planteamientos casuísticos, procedimentalistas, kantianos, utilitaristas, comunitaristas y desde las éticas del cuidado y de las virtudes (Toulmin, 1981; Clouser \& Gert, 1990; Rubinstein, 1996; Elliot, 1998; Callahan, 2003; Harris, 2003). Por este motivo diversos autores afines al principialismo, como Gillon \& Lloyd (1996) y J. C. Siurana (2010), han reconocido la necesidad de explorar su complementación con otros enfoques, con la finalidad de afinar su pertinencia cultural y evitar el universalismo acrítico en su aplicación.

Estas observaciones han motivado un creciente interés por los enfoques narrativos en bioética, lo que ha llevado a la creación de revistas especializadas, como Narrative Inquiry in Bioethics, y a la realización de congresos internacionales, en el marco de las asociaciones académicas de humanidades médicas. Por esa razón T. Domingo Moratalla y L. Feito (2013: 15) sostienen que ya es posible hablar de la "bioética narrativa" como un enfoque propio, complementario al principialismo, que permitiría atender a los factores experienciales y contextuales inherentes a las éticas de la vida. Su punto de partida sería una teoría consensual de la verdad, basada en el "realismo crítico del sentido". O en palabras de Arras:

El campo de la bioética está empezando a tomar su propio giro narrativo. Largo tiempo dominada por las aspiraciones a la objetividad y la universalidad que se concreta en su paradigma dominante "principialista", la bioética está siendo testigo de una explosión de interés por la narrativa y la narración como formas alternativas de estructuración y evaluación de las experiencias de los pacientes, médicos, y otros profesionales de la salud (Arras, 1997: 66).

Este "giro narrativo" permitiría una apertura epistemológica, situando el proceso ético-deliberativo dentro de una historia abierta, donde "lo que se es" se entiende a partir del estar "entre los otros" (Ricoeur, 1996: 185). O en otros términos, desde un "sí mismo" que presupone al sujeto autónomo, pero que sólo se entiende a plenitud en la relacionalidad permanente de la alteridad. Usando el modelo ricoeriano para describir esta tensión, comprendemos que no es posible concebir a un sujeto separado, de forma absoluta e independiente de los otros y del mundo, pero tampoco a un sujeto autónomo que se disuelve en los demás, y que se pierde en la impersonal colectividad.

Las bases teóricas y metodológicas que permiten fundamentar este nuevo enfoque son heterogéneas, pero comparten un interés común por ampliar los criterios interpretativos de las éticas de la vida. Cabe situar 
entre ellas a la "ética del discurso", propuesta por Karl-Otto Apel en colaboración con Jürgen Habermas. Se trata, en este caso, de una síntesis original entre la tradición kantiana y el pragmaticismo de C. S. Peirce, por medio de pragmática trascendental del lenguaje, que responde a la dimensión dialógica de la racionalidad intersubjetiva.

Como observa Adela Cortina (1994), la ética dialógica apeliana se orienta ante todo a la implementación de "sistemas de gestión ética", aplicables a nivel meso y macro institucional. Ello no obsta para reconocer que las éticas del discurso no siempre muestran el mismo grado de pertinencia práctica en el plano de los dilemas micro-sociales y ante dilemas en los que se precisa de un juicio prudencial personalizado, similares a los que se dan en el caso de la práctica clínica y en la relación médico-paciente. En el nivel interpersonal, y desde las percepciones propias del mundo de la vida, resulta fecundo el recurso a las corrientes hermenéuticas a las éticas aplicadas, que aportan pautas deliberativas distintas a las que se desprenden a partir de enfoques basados en normas abstractas, de matriz cognitivista, ya que no buscan dictar normas o reglas, sino enunciar las condiciones de posibilidad en el proceso de comprensión.

Es en este plano hermeneutizable donde se debe hacer uso de autores como H.G. Gadamer (2007) y P. Ricoeur los que permiten inducir una "ética hermenéutica" (Conill, 2006) que entroncando con la phronesis aristotélica, valorice la trama identitaria narrativo-biográfica de la persona, estableciendo mediaciones analíticas desde el nivel prudencial, hasta los niveles deontológicos y reflexivos.

En el caso del modelo ricoeuriano se percibe un intento deliberado de enlazar distintas perspectivas, como la "ética de la virtud" de Alasdair MacIntyre (1981), que aprecia las tradiciones culturales en tanto procesos de transmisión narrativa, las indagaciones analíticas de Bernard Williams (1987) que estudia los relatos sobre la "vida digna de ser vivida", la ética reconstructiva de Jean-Marc Ferry (1996) que integra "argumentación" con "narración".

En particular se debe reseñar el trabajo de Martha Nussbaum (1995; 2013), orientado al diálogo entre literatura y filosofía, a partir de una teoría de la "imaginación narrativa", entendida como capacidad de interacción moral en clave cosmopolita. En Nussbaum resulta importante destacar que su ideal eudaimonista del "florecimiento humano" permite integrar las dimensiones filosóficas y las literario-narrativas, ya que muestra sstemáticamente porqué las preguntas referidas a la elección humana y la acción, las cuestiones estéticas, y las preguntas morales no pueden ser abordadas en departamentos estancos.

Otro punto de partida para una "ética hermenéutica" se podría situar en la fenomenología y en la teoría diltheyana del conocimiento, las que 
aportan las bases de una "filosofía narrativa". Esta idea fue recogida explícitamente en la propuesta de Wilhelm Schapp (1953, 1959) cuya obra interpreta la vida como capacidad de establecer "historias entrelazadas" (Geschichten Verstricket), que más allá de la descripción y la prescripción, permiten narrar un fondo moral valorativo. Con Schapp también podemos realizar una relectura de la "hermenéutica de la corporalidad" de Helmut Plessner (1928), basada en la distinción entre "tener un cuerpo" (Körper Haben) y "estar en un cuerpo" (Leib Sein), lo que lleva a complementar el a priori de la conciencia por medio de un a priori corporal, basado en la razón experiencial.

Esta aproximación antropológica se puede complementar desde la filosofía política de Hannah Arendt y su "teoría de la acción" que proyecta una metodología deliberativa que articula narrativamente la tensión entre igualdad y diferencia, a partir de la identidad biográfica, entendida como categoría interpretativa. En La Condición humana Arendt cita a la escritora danesa Isak Dinesen para resaltar expresamente las propiedades terapéuticas de la narratividad humana: "Se puede soportar todo el dolor si se lo pone en una historia o se cuenta una historia de él" (Arendt: 1993: 199).

La filósofa entiende que toda vida individual, entre el nacimiento y la muerte, puede contarse finalmente como una narración, con comienzo y fin, en tanto condición prepolítica y pre histórica de la "Historia", entendida como gran narración sin comienzo ni fin que incluye a toda la humanidad (Arendt, 1993: 208). Ella observa en su teoría de la Acción que "la esfera de los asuntos humanos está formada por la trama de las relaciones humanas que existe donde quiera que lo hombres viven juntos" (Arendt, 1993: 207). En esta trama la acción humana "produce historias con o sin in tención de manera tan natural como la fabricación produce cosas tangibles" (Arendt, 1993: 208). Pero en esas historias "nadie es autor o productor de la historia de su propia vida" (Arendt, 1993: 208).

El carácter pre-político de estas narraciones radica en que se es actor o paciente de ellas, pero nunca su autor, ya que la autoría propiamente tal es de la humanidad como un todo, entendida como un agente abstracto, distinto al "héroe" de las narraciones, que ejerce su rol como los recitadores que re-interpreran el argumento de la obra y en ese proceso son capaces de trasmitir su pleno significado (Arendt, 1993: 2011).

Siguiendo estas aproximaciones interpretativas la bioética narrativa opera como una teleología de los actos humanos, entendidas como conexiones entitativas, impregnadas de intuiciones axiológicas. En ese proceso la hermenéutica no cabe a un sujeto aislado y desvinculado, sino a un colectivo humano en proceso de continua ampliación. En el "gran teatro del mundo", usando la expresión de Calderón, las historias indivi- 
duales no pueden permanecer en el solipsismo de la conciencia personal, "ya que la acción y el discurso necesitan la presencia de otros no menos que la fabricación requiere la presencia de la naturaleza para su material" (Arendt, 1993: 211).

\section{Desde las claves de la medicina narrativa}

Desde el plano metodológico la bioética narrativa asume las contribuciones de la "medicina narrativa", entendida como un encuadre de la práctica clínica que intenta superar la simple compilación de datos y pruebas para incorporar el diálogo, la empatía y la comprensión profunda de los pacientes. Se entiende que cada persona concurre ante el personal sanitario portando el gran relato de su propia vida, en el cual la enfermedad constituye el último cuadro de una puesta en escena. Sólo una mirada integral a toda esa historia podrá aportar un sentido auténtico a una narración compleja, que es imposible de entender desgajada en escenas aisladas, o dispersa en fragmentos y síntomas sin organizar.

Esta intuición presupone que la palabra narrada posee un valor terapéutico en tanto la experiencia de enfermedad demanda un momento de exteriorización, que dé inicio a la búsqueda de su superación. En $\mathrm{La}$ Celestina Fernando de Rojas aporta un diálogo muy ilustrativo de esta dimensión al hacer decir a Pleberio a su hija Melibea:

Hija, mi bien amada y querida del viejo padre, por Dios, no te ponga desesperación el cruel tormento de esta tu enfermedad y pasión, que a los flacos corazones el dolor los arguye. Si tú me cuentas tu mal, luego será remediado. Que ni faltarán medicinas ni médicos ni sirvientes para buscar tu salud, ahora consista en hierbas o en piedras o en palabras o esté secreta en cuerpos de animales. Pues no me fatigues más, no me atormentes, no me hagas salir de mi seso y dime ¿qué sientes? (De Rojas, 2006: 177).

Esta propuesta se ha sistematizado desde distintos autores, entre los que cabe destacar a Rita Charon (1993, 1994, 2001, 2004), Martha Montello (1995) y Kathryn Montgomery (1991, 2006), todas académicas ligadas al desarrollo de las humanidades médicas. Su interés se puede entender como un movimiento de renovación de las prácticas clínicas que busca cualificar los procesos de comunicación en la atención sanitaria, ensanchando las fuentes de razonamiento y aprendizaje, posibilitando la adquisición de "competencias narrativas". Para eso revaloriza los aspectos relacionales y comunicativos, propios del ejercicio clásico de la medicina, olvidados durante el siglo XX debido al predominio de enfoques exclusivamente biomédicos y tecnológicos. Se utiliza para ello distintas 
metodologías cualitativas, como diarios clínicos, entre otros tipos de registro.

También se puede afirmar que la bioética narrativa hereda los aportes de la psicología constructivista de Jerome Bruner (1991). Su obra colabora a clarificar la diferencia entre "pensamiento paradigmático", orientado a la conceptualización y categorización, y el "pensamiento narrativo", encaminado a expresar la intencionalidad de la acción humana. Esta distinción permite situar epistemológicamente la narratividad como un ámbito de deliberación moral.

\section{La razón narrativa y la tradición racio-vitalista}

A su vez el desarrollo de la medicina narrativa ha permitido revalorizar aspectos de la tradición filosófica orteguiana, entendiendo que un "racio-vitalismo narrativo" puede aportar perspectivas fecundas a la atención sociosanitaria y bioética. Al respecto conviene recordar que el propio Ortega planteaba que:

Frente a la razón pura físico-matemática hay, pues, una razón narrativa. Para comprender algo humano, personal o colectivo, hay que contar una historia. Este hombre, esta nación, hace tal cosa y es así porque antes hizo tal otra y fue de tal otro modo. La vida sólo se vuelve algo transparente ante la razón histórica (Ortega y Gasset 1975: 5).

El desarrollo de la obra y perspectiva de Ortega ha sido continuada por una pléyade de autores que en sus diversos énfasis y estilos han profundizado el enfoque racio-vitalista. En esa tradición podemos situar a P. Laín Entralgo, Julián Marías, Xavier Zubiri, Diego Gracia y a partir de sus últimas obras, a Adela Cortina. Todos estos autores contribuyen a la concreción de una reflexión bioética en perspectiva narrativa y hermenéutica.

Laín expone sistemáticamente su valorización de la narratividad clínica en La relación médico enfermo, obra que presupone que "el diálogo entre el médico y el enfermo es a la vez y siempre diagnóstico y terapéutico" (Laín Entralgo 1964: 307). De allí que Laín sintetice su valoración de esta dimensión afirmando:

Cuando el enfermo en cuanto tal enfermo, habla al médico, le llama, le notifica algo, nombra con precisión mayor o menor lo notificado y le persuade, disuade o contrasuade; y, por otra parte, da y recibe compañía (la recibe, esto es lo decisivo), logra cierto sosiego interior, se esclarece íntimamente a sí mismo y, en cierto modo (a veces con orgullo de enfermo) se 
autoafirma, vive la importancia de su propia persona (Laín Entralgo 1964: 311).

El doctor Laín Entralgo atribuye de esta forma al individuo tres modos de operación: como "autor y paciente de sí mismo", "actor" y "espectador" de su vida, y a la vez "intérprete o hermeneuta" de sí, "autopoeta" de su condición humana (Laín Entralgo 1964: 318).

También en abierta continuidad a este enfoque racio-vitalista, la obra de Julián Marías es un aporte la filosofía de la convivencialidad y la concordia, que sin reprimir el desacuerdo o el conflicto, busca que la discrepancia no impida la vida en común (Marías 2000). A la vez, Marías despliega el perspectivismo de Ortega al pensar la vida humana como un proceso sistemático en el cual la libertad es indelegable e irrenunciable:

El hombre tiene que elegir en cada instante lo que va a hacer, lo que va a ser. ¿Cómo? Se encuentra con la circunstancia, con todo lo que lo rodea, desde su cuerpo y su psique hasta las galaxias y lo trascendente, en la medida en que de una u otra manera lo halla en su horizonte. El hombre no elige 10 que es, sino quién va a ser. Su proyecto vital —o cada uno de los proyectos particulares en que se articula y realiza - se proyecta sobre la circunstancia y la convierte así en un repertorio de posibilidades (o imposibilidades). Entre ellas, el hombre prefiere una u otra, e intenta realizarla (Marías, 1984: 4).

De esa forma la relacionalidad de la libertad humana resulta evidente en tanto "las libertades, por su carácter sistemático, fundado en el de la vida humana, funcionan en contexto. Las otras libertades, que ciertamente restringen cada una de las singulares, son las que le dan fuerza para poder realizarse" (Marías, 1984: 6). De allí que el proyecto vital de las personas exija una búsqueda de aquella "fusión de horizontes" (gadameriana) que sólo se logra en la reciprocidad y el reconocimiento comunicativo de la alteridad.

También se puede entrar en la racionalidad narrativa desde la inteligencia "sentiente" y la razón "sensible", tal como lo propone Xavier Zubiri. Para el filósofo vasco el acto de sentir implica también un conocimiento, una gnosis. Si para los racionalismos lo "sensible" es mero dato o un conjunto de intuiciones, para Zubiri no hay dos actos separables: uno, de sentir, y otro, de inteligir, sino un solo acto para un mismo objeto (Zubiri, 1966:341). Se trata de una apertura conceptual y epistemológica que posee consecuencias para comprensión intelectual. Adquiere importancia un principio corporal y emotivo, que el relacionarse ante el otro, sin juicios ni indiferencia, requiere una voluntaria actitud de indefinición que nace de aprehender la realidad sin juzgarla ni relativizarla. 
En la obra de Diego Gracia se debe reconocer la fundamentación de una "bioética deliberativa", que existe y se desarrolla de forma cotidiana como una "una escuela de deliberación" (Gracia, 2002a: 37). De esa forma la reflexión bioética es arrancada de las circunspectas especializaciones clínicas para abrirse a la palabra universal:

Ya no pueden ser los médicos, ni los políticos, ni los economistas, ni tampoco los sacerdotes o los teólogos quienes detenten el monopolio de la decisión en este tipo de cuestiones. Ha de ser la sociedad entera la que delibere y decida sobre ellas (Gracia, 2002a: 39).

Entramos de esta forma en lo que Gracia llama un tercer momento en la historia de la exploración humana de la realidad que se debería entender como una "edad hermenéutica de la razón" (Gracia, 2002b: 188). Por lo cual la bioética debe evolucionar: "de ser una disciplina fundamentalmente clínica está pasando paulatinamente a convertirse en un instrumento de análisis social, institucional y político" (Gracia, 2002a: 38).

Finalmente podemos situar a Adela Cortina como una última autora clave que expresa de un modo singular la intuición central del enfoque racio-vitalista inaugurado por Ortega. Esta sintonía se puede apreciar de forma más clara en algunas de sus obras más recientes, que intentan conjugar las éticas de matriz kantiana y procedimentalistas con la dimensión afectiva del ser humano. De allí su interés por fundamentar una ethica cordis (2007) que atienda a que todo acto comunicativo no sólo contempla una dimensión discursiva y argumentativa, sino que también un aspecto cordial y compasivo, inherente a la comunicación total del ser humano. Encuentra un punto de anclaje a esta integración en la categoría de "corazón" comprendido de forma integral: "Cordis es sin duda afecto, pero también inteligencia, talento, espíritu, incluso estómago, porque hay que tener estómago -y mucho- para ser justo" (Cortina, 2007: 193). La propuesta de Cortina es relevante en tanto busca superar la distinción tradicional entre éticas formales y materiales, deontológicas y teleológicas, cognitivistas y decisionistas, para explorar en la idea de "reconocimiento recíproco" el fundamento de una ética cívica que haga de la comunicación intersubjetiva un punto de articulación que no anule el pluralismo moral de nuestra época. 


\section{Aportes de la antropología médica y los enfoques interculturales en bioética}

Por otra parte, en la medida en que se ha desarrollado la antropología médica ha sido posible ampliar la comprensión del origen social de la enfermedad. Esa constatación es relevante para la fundamentación del proyecto de la bioética narrativa en la medida en que los aspectos cualitativos que la antropología logra estudiar, mediante prácticas de observación participante y métodos etnográficos, dan cuenta de las especificidades de distintas culturas terapéuticas, tradiciones medicinales y sistemas biomédicos.

La primera consecuencia de esta relación conceptual radica en poner en primer plano la necesidad de pertinencia intercultural que demanda el debate bioético. Al respecto cabe reseñar los aportes de la etnología médica de E. H. Ackerknecht, quién mostró que la llamada "medicina primitiva" no se debe entender como el embrión directo de la medicina moderna, ya que debe estudiarse en sí misma, en cuanto dispositivo cultural, excede los límites de los procesos biomédicos. De esa forma Ackerknecht distingue entre la "medicina primitiva" y una "medicina popular", que dando continuidad a prácticas heredadas de la primera, incorpora y sintetiza elementos aportados por la "medicina galénica" (Ackerknecht, 1985: 9). De allí que todo análisis de la práctica médica deba tender a reconocer el pluralismo epistemológico que subyace a sus prácticas, las cuales demandan marcos interpretativos específicos. Este análisis coincide con la antropología simbólica de Clifford Geerz (1973) y su método de "descripción densa" que intenta interpretar las representaciones narrativas por las cuales se configuran las culturas.

La antropología médica ha impulsado el desarrollo de una mirada intercultural en bioética. Al respecto cabe remitirse a la obra de Raúl Fornet Betancourt (2001), quién propuso una Transformación intercultural de la filosofía mediante una hermenéutica pluritópica que busque "desontologizar las culturas". La propuesta intercultural busca una "reubicación de la razón”, que amplíe las fuentes de entendimiento e interpretación de los sujetos. Se trata a su juicio de:

Un proceso de aprendizaje intercultural que amplía los criterios de discernimiento y compensa las unilateralidades de la razón en su figura histórica de razón sometida a la dinámica del desarrollo cultural-civilizatorio de la modernidad occidentalcapitalista (Fornet-Betancourt, 2005: 404).

Esta comprensión demanda una hermenéutica de la alteridad 
que parte del reconocimiento del extraño como interprete y traductor de su propia identidad, que supera el horizonte de la división sujeto-objeto y hace del trabajo hermenéutico un proceso de intercambio de interpretaciones (Fornet-Betancourt, 2005: 406).

Este proceso hermenéutico intercultural debe ser aplicado a las preguntas y desafíos de la bioética narrativa y puede aportar a la superación de los conflictos entre distintas prácticas médicas y sanitarias, y superar el debate entre concepciones bioéticas ancladas en concepciones paternalistas y las perspectivas actuales de la bioética de los principios. Se trata de un debate que posee especial pertinencia en un contexto de contraste entre una modernización económica y tecnológica acelerada y un malestar intersubjetivo, documentado cada vez más ampliamente (Lechner, 2002).

\section{Salud intercultural y complementariedad de sistemas médicos}

El acervo de la bioética narrativa, expresado y fundamentado en el amplio corpus bibliográfico que se ha reseñado, podría colaborar a la composición de una ética de la vida adecuada a los sistemas de salud que se insertan en relaciones de tipo intercultural (Tealdi, 2008). En palabras de Buxó Rey (2004: 15) un enfoque así permitiría el reconocimiento de la diversidad situacional y contextual como inicio de toda discusión, negociación y acuerdo entre distintas comunidades tecnocientíficas, culturales y morales, e impedir la clausura discursiva y el solipsismo metódico.

Una de las más experiencias prácticas que han buscado des-centrar la razón occidental para abrirse a la alteridad radical del saber ancestral indígena ha sido la institucionalización de sistemas de "salud intercultural". Esta idea ya fue propuesta en los años cincuenta por G. Aguirre Beltrán (1995) pero sólo en los últimos veinte años se han comenzado a implementar de forma sistemática bajo la forma de programas interculturales en las instituciones de salud primaria. Este cambio se debió a un nuevo énfasis en las prioridades de la Organización Panamericana de la Salud (Dion Stout y Coloma, 993).

Además, cabe recordar la influencia de la "declaración de Naciones Unidas sobre los Pueblos Indígenas y su derecho a la salud" (2007), la importancia del Convenio No 169 de la OIT, y el reconocimiento de UNESCO a la medicina Kallawaya (Bolivia) como patrimonio intangible e inmaterial de los pueblos, en 2008. Hoy varios países andinos han otorgado reconocimiento constitucional a sus políticas de salud intercultural y su implementación está encomendada a autoridades con rango viceministerial. Sin embargo, investigadores como Cuyul (2013), sostienen que 
junto a los aspectos valorables de estos nuevos proyectos, en el plano experiencial lo que aparece es una forma de tolerancia pasiva a las prácticas fundadas en la sabiduría ancestral, más que una dinámica en la cual ambas racionalidades culturales, indígena y occidental, estén desafiadas a reposicionarse en un encuentro crítico y creativo, de carácter integrador.

Según Fernández Juárez (2011) la institucionalización de los programas interculturales en salud se explica por una reacción de los Estados ante la desconfianza que manifestaron muchas comunidades indígenas ante ciertas prácticas biomédicas, en especial ante los procedimientos quirúrgicos, programas de vacunación masiva, internado hospitalario, $\mathrm{y}$ medicalización del embarazo y el parto. En especial, se reaccionó con fuerza ante las políticas masivas de esterilización de mujeres indígenas sin consentimiento informado, que en los casos de Perú y México se mantuvieron hasta mediados de la década del noventa (Menéndez, 2009; Málaga, 2013).

Los procesos de interculturación en salud exigen argumentos y métodos más consistentes, con la finalidad de evitar la duplicidad sistémica y la tolerancia pasiva que denuncia Cuyul. Para poder hablar en estricto rigor de salud intercultural se debería lograr que las diferencias culturales se expresen, explícita o implícitamente, en los vínculos intersubjetivos. Por lo tanto, no basta la simple interacción pasiva entre individuos, situados en marcos culturales diferenciados. Se requiere la implicación activa de la desemejanza cultural. En palabras de Denoux la inter-cultura es el intento de "metabolizar las diferencias") para arribar a una síntesis entre las partes intervinientes (Denoux, 1994: 72). Bajo estos criterios un enfoque en salud intercultural se debería entender como:

La práctica y el proceso relacional que se establecen entre el personal de salud y los enfermos, donde ambos pertenecen a culturas diferentes, y donde se requiere de un recíproco entendimiento para que los resultados del contacto (consulta, intervención, consejería) sean satisfactorios para las dos partes (Campos Navarro, 2004: 129).

\section{Dilemas bioéticos en el sistema salud intercultural chileno}

En Chile la discusión pública sobre salud intercultural se inició en 1996, en el marco del "Primer encuentro nacional salud y pueblos indígenas" (OPS, 1996). Este acercamiento del Estado reflejó un desplazamiento en el análisis de las deficiencias e inequidades en la atención socio-sanitaria de la población indígena, revalorándose los factores subjetivos en los procesos terapéuticos, caracterizados como mecanismos de 
legitimación cultural, que permiten a los pacientes aceptar las causas de su enfermedad, y observar adecuadamente los tratamientos prescritos.

Hoy, luego de una primera fase de implementación piloto, el debate se ha centrado la necesaria complementación entre las experiencias de salud basadas en la sabiduría indígena y las prácticas biomédicas. Según Álvarez (2007) en los hechos estos sistemas se relacionan de tres posibles maneras: como "incorporación formal de un enfoque intercultural al interior del sistema de salud oficial, ya sea hospitalario, o en la atención primaria", como "aplicación de hecho por parte de funcionarios indígenas de tratamientos herbolarios, a espaldas de las autoridades de los establecimientos de salud", o como "readecuación de los recursos de los sistemas locales en salud, de los que dispone la población" en orden a una complementariedad integral de los modelos médicos en todas las fases del proceso de salud/enfermedad (Álvarez, 2007: 3). De acuerdo a esta clasificación el autor advierte que predominan las dos primeras modalidades, las que revelan procesos de coexistencia paralela y tolerancia pasiva. De allí que concluya que a la fecha "no se ha logrado consolidar la complementariedad de sistemas médicos" (Álvarez, 2007: 213).

Para el investigador mapuche Andrés Cuyul los principales nudos de conflicto que actualmente atraviesan la relación entre los sistemas de salud occidental-galénico y las instituciones terapéuticas mapuche se expresan en los siguientes puntos:

i) los agentes de salud mapuche al interior de los servicios son condicionados en su accionar por el sistema de salud chileno; ii) las prácticas de salud mapuche se estandarizan, codifican, valorizan y notifican, al igual que las prestaciones de salud wingka; iii) la lógica de retribución por el bien (mañuntu) cambia a una racionalidad neoliberal; iv) el contexto/escenario de sanación en el territorio cambia al box de atención, aislado de la realidad y variables que hacen a la salud y a la enfermedad, al igual que en la clínica médica; v) las acciones en salud mapuche pasan a formar parte del patrimonio y capital del sistema de salud chileno; y, en definitiva, vi) se determina la reproducción de agentes de salud mapuche a la contratación y pago de parte del Estado chileno y su funcionamiento en box de atención, ya no en el territorio. Progresivamente se interviene la cultural misma, determinándola y condicionándola mediante el dinero del Estado (Cuyul, 2013: 7).

Desde el punto de vista del Estado también se aprecian tensiones y conflictos similares. Según el estudio "Sistematización de las experiencias de salud intercultural" (MIDEPLAN, 2010) los conflictos de interpretación entre los dos modelos de salud se expresan forma indirecta como "dificultades en la interacción, confianza y comunicación con médicos, personal y funcionarios de la salud pública” (MIDEPLAN, 2011: 48) y 
"carencia de normativas que respeten las particularidad de las culturas indígenas" (MIDEPLAN, 2011: 210).

Salaverry entiende que la perspectiva intercultural aún se percibe como una "categoría en construcción, que causa confusión en los profesionales de la salud" (Salaverry, 2010: 93) debido a que su institucionalización no respondió a una reflexión del cuerpo médico, sino a una respuesta de los Estados a las demandas indígenas. Pero Fernández Juárez (2011) matiza este juicio al advertir que las "posturas radicales de exclusión no son inhabituales, ni desde la óptica de la biomedicina, ni tampoco desde las medicinas originarias". De allí que la falta de complementariedad no sólo se explique por carencias en la formación del personal médico y hospitalario en áreas atingentes al reconocimiento intercultural, o por fallas en los procedimientos funcionales y en los marcos jurídicos, sino también por insuficiencias a nivel de la fundamentación conceptual y procedimental, debido a un desfase epistemológico.

Tras la aceptación de algunas limitadas características y tecnologías de la medicina tradicional, "persiste un rechazo más o menos global a las bases ontológicas de la medicina indígena" (Álvarez, 2007: 3). La interacción cultural entre ambos sistemas sanitarios no sólo pide implementar procedimientos funcionales y legales adecuados, sino también estrategias de reconocimiento de su alteridad valorativa y la elaboración de criterios hermenéuticos que faciliten su reconocimiento recíproco.

En el plano de la deliberación bioética esta dificultad se expresa de una forma directa. Por una parte, los "principios de Georgetown" proponen una noción de "autonomía" como capacidad de actuar libremente, de acuerdo con un plan autoescogido, sin coacción externa, y bajo garantías que permitan ejercer la capacidad de agencia y la acción intencional. En abierto contraste, la sabiduría ancestral mapuche valoriza los equilibrios en las relaciones comunitarias, que mandatan vínculos relacionales, fundados en la interdependencia. De allí la necesidad de una bioética narrativa, en clave intercultural, que facilite los procesos de esclarecimiento e interpretación de los conceptos y valoraciones morales en estos contextos.

\section{La sabiduría ancestral mapuche y sus formas narrativas}

Expuesta la realidad compleja y desafiante de la salud intercultural resulta evidente que el enfoque bioético narrativo es particularmente importante a la hora de estudiar la realidad de la salud intercultural en el marco de las comunidades mapuche.

Su desarrollo puede facilitar la integración de los sistemas biomédicos tensionados por contextos marcados por la desigualdad y la asime- 
tría, como los que se dan en Chile entre la sociedad mapuche y la sociedad chilena-occidental (Citarella et al., 1995: 32). La relevancia de programa de investigación de esta naturaleza se advierte al constatar que la sabiduría (Kimün) del pueblo mapuche no sólo propone una comprensión y explicación del mundo de la vida en un nivel epistémológico (Curivil, 2008). Más radicalmente, el «pensar mapuche» (Rakiduam) se despliega en el acto mismo de narrar (Marileo, 1995). Aplicando a ese proceso una hermenéutica y una pragmática de la cultura, se observa que su racionalidad constituye "una trama de sentidos y significados que se transmiten por medio de símbolos, mitos, dichos, relatos, prácticas y reconstrucciones" (Salas, 2003: 55), que manifiestan narrativamente el sentido de la existencia de los sujetos, como autocomprensión y héterocomprensión a la vez.

De allí que sus formas narrativas constituyan el núcleo semiótico de su experiencia vital, que se articula por medio de códigos de comportamiento y "normas de uso", arraigadas a un modus vivendi, transmitido mediante lazos tradicionales e intergeneracionales, que vinculan mutuamente a los individuos y los singularizan de cara a su entorno social y natural. La narratividad otorga dinamicidad a estos sistemas normativos, abriendo la posibilidad de re-significarlos creativamente.

La sabiduría mapuche denomina a este proceso de adhesión subjetiva feyentun, lo que se puede traducir como obediencia, creencia, convicción o adhesión a un corpus de creencias normas de uso y tradiciones rituales heredadas de los antepasados ( $A d M a p u)$, que definen las relaciones con el "otro-en cuanto prójimo" (ka ce o ka xipace), entendido como viviente de esta tierra. Quienes pueden ser considerados ka ce o ka xipace pueden variar, implicando una mayor universalidad o restricción normativa (Curivil, 2008). Se trata de un reconocimiento intersubjetivo basado en las convicciones propias del feyentun.

Ricardo Salas (1996) distingue un doble nivel en las formas narrativas mapuches que transmiten el $A d M a p u$. Por una parte identifica los relatos Epeu, que cumplen funciones didácticas de tipo moral, y por otra los relatos Nütram, que expresan la propia identidad histórico-biográfica, en un marco mítico-filosófico y teológico. Ambos niveles poseen sus propias razones o criterios veritativos y sus modos de validación éticos y normativos, de los cuales emana una determinada "eficacia simbólica" (Levi Strauss, 1968: 168). Ello explica que sus prácticas tradicionales de salud contemplen tres niveles mutuamente integrados: preventivo, curativo, y ritual (OPS, 2008: 35).

En coherencia con este paradigma, la racionalidad vital mapuche comprende la enfermedad (Küxan) "como la pérdida de equilibrio de las relaciones ser humano-espíritu-naturaleza, y una transgresión de normas 
y principios de convivencia" (OPS, 2008: 33). Y por contraste, la salud se expresa como Küme Felen (estar bien), entendida como un bienestar que supera la dimensión biológica individual, ya que considera factores de orden social, ambiental y espiritual. Se "está bien" en tanto se atiende al «buen vivir» (Küme Mongen), entendido como una vida deseable, fundada en una noción ampliada de la dignidad, que integra armónicamente al ser humano y a la naturaleza. Un observador de estas prácticas describe la forma en que se concretiza esta relacionaldad cósmica:

Cuando una Machi (shamana Mapuche) acude al bosque a buscar sus hierbas medicinales, deja en el lugar de la extracción pequeños cantaritos o "metawes", o trozos de lanas de colores. Con esta actitud está devolviendo algo a la tierra, en reconocimiento de lo que ha tomado gratuitamente de ella. Este vínculo con la madre tierra es la expresión de la reciprocidad como una actitud cósmica. Por otra parte, la Cosmovisón Mapuche de manera similar a como sucede en otras cosmovisones originarias, plantea la interrelación de todo con todo. Es la trama universal donde todo se vincula con todo (Sodepaz-Pachakuti, 2004: 1).

Esta compleja trama no se puede comprender desde una racionalidad monológica, que niegue la reflexividad de los modos de vida y los saberes culturales ancestrales, al catalogarlos bajo primas ahistóricos, atemporales, abstractos y simplificadores. El reconocimiento de la experiencia vital de los sujetos que se autocomprenden narrativamente como Mapuche demanda un horizonte hermenéutico en el cual se confronten los saberes culturales y las racionalidades teleológicas, de modo que sus expresiones narrativas puedan reconocerse como portadoras de un nivel de verdad al que se accede contextualizada y relacionalmente, en jun plano distinto y diferenciado al nivel estrictamente epistemológico. Al respecto cabe recordar la función social que Durkheim atribuye a la religión, en un plano esencialmente moral, y no cognoscitivo:

La verdadera función de la religión no es hacernos pensar, enriquecer nuestro conocimiento, agregar a las representaciones que obtenemos de la ciencia representaciones que tiene otro origen y otras características, sino hacernos actuar, ayudarnos a vivir (Durkheim, 1992: 338).

\section{Conclusiones}

En contextos de conflicto y asimetría, como los que caracterizan los territorios en los que habita el pueblo mapuche, y en los que operan convicciones arraigadas, surgidas de experiencias históricas, pueden enfrentarse a controversias que sin ser irreductibles, son de compleja su- 
peración. No basta tolerar el pensamiento extraño, ya que sin el reconocimiento de alteridad legítima es imposible abrirse a un pensamiento verdaderamente universal.

La integración o complementación de los sistemas de salud basados en paradigmas culturales diferenciados representa un reto de nuestro tiempo. En Chile este desafío se concretiza en la posibilidad de legitimar y cualificar las prácticas interculturales en salud mapuche. Esta tarea no se puede resolver en un orden procedimental o institucional. Se requiere profundizar en las bases conceptuales que subyacen y distinguen a cada sistema biomédico.

En el caso de la medicina galénica occidental se requiere ampliar el criterio que fija en el individualismo posesivo un axioma interpretativo basal, con una pretensión universalizante. Al respecto cabe la observación levinasiana que afirma: "Para la tradición filosófica de Occidente, toda relación entre el Mismo y lo Otro, cuando no es ya la afirmación de la supremacía del Mismo, se remite a una relación impersonal en el orden universal" (Lévinas, 1997: 110).

La bioética narrativa, sin abandonar las bases de la tradición del pensamiento occidental aporta a esta tarea un fundamento relevante y pertinente. En cierta forma su reflexión y puesta en práctica actualiza las observaciones de Ricoeur al sostener que todo acto ético es un momento hermenéutico: "La intencionalidad ética es siempre una acto hermenéutico en el que nos entendemos en una historia, por lo que «en el plano ético, la interpretación de sí se convierte en estima de sí" (Ricoeur, 1996: 176).

Bajo la racionalidad narrativa el yo del sujeto autónomo sólo se entiende cuando está “entre los otros”. Esta constatación determina que las instituciones justas, en las que es posible la intencionalidad de vida buena, se debe concebir como un espacios relacional, en los que sea posible "tomar parte" en conversaciones que den sentido a la propia identidad narrativa, tal como lo expresa Haker:

La hermenéutica de la ética apunta tanto a la conexión de la moralidad con identidad, así como con la experiencia. La experiencia ética de tener siempre responsabilidad no se puede ver de forma independiente de la propia historia de vida individual, que a su vez está conectada de muchas maneras diferentes, con aspectos sociales, históricos, culturales y políticos (Haker 2006: 355).

Las experiencias propuestas por la naciente bioética narrativa, entendida como un proceso de deliberación intersubjetiva, se muestra como un posible campo de experimentación en el proceso de integrar intercul- 
turalmente la perspectiva teleológica de la cosmovisión mapuche, expresada en la búsqueda mapuche del "buen vivir" (Küme Mongen), y el imperativo deontológico de "justicia para todos", fundamentado en la bioética principialista, y más ampliamente, en la Declaración Universal de los Derechos Humanos.

De esta forma el enfoque narrativo puede colaborar a la resignificación de las prácticas de la salud intercultural mapuche, posibilitando su integración crítica en el sistema general de salud, sin menoscabo a su propia singularidad. Para ello debe lograr construir un marco criteriológico que colabore a que ambos componentes del sistema de salud, tanto el biomédico como el intercultural, incrementen sus aprendizajes, deliberen de forma sistemática y arriben a decisiones responsables, a partir de la crítica recíproca a sus propias pautas bioéticas.

Este proceso debe decantar en un proceso de traducción intercultural que garantice una radical apertura al otro, como depositario de razones que van más allá de los prejuicios y supuestos que puedan anidar en una determinada tradición terapéutica. No se trata solamente de criterios procedimentales, como los de la ética del discurso, (que por lo demás se asumen como un supuesto básico). Además se requiere un principio hermenéutico que colabore en enmendar las aporías de un planteamiento de la autonomía que puede llevar a legitimar la ruptura de los vínculos en los que todo ser humano realiza su proyecto de vida buena.

El logro de la integración de paradigmas y racionalidades biomédicas debería concretarse en medidas y políticas de salud distintas a las actuales. Entre otras orientaciones, se debería explorar la co-gestión de los centros de salud intercultural mapuche, involucrando a la comunidad circundante, mediante espacios deliberativos diseñados específicamente para estos efectos. En este nivel es necesario evitar la esencialización de las prácticas de salud mapuche, reduciéndolas a estereotipos o atavismos arcaicos, para descubrir su fondo simbólico-interpretativo, que puede evolucionar críticamente.

A nivel macro institucional se hace necesaria una Subsecretaría de Salud Intercultural o Indígena, u otra instancia de alto rango gubernamental, que elabore una política integrada y a escala nacional que reforme los programas de salud intercultural que se implementan hoy en territorios indígenas, y que permita avanzar de forma sistemática en la armonización de las prácticas sanitarias.

Otro aspecto crucial es la comprensión de la naturaleza abierta del conocimiento ancestral mapuche, para evitar su commoditización por medio de patentes y derechos de autor que clausuran el uso de procesos y productos terapéuticos, y que niegan la perspectiva de derechos colectivos, propia de la cosmovisión indígena. Finalmente se requeriría la instaura- 
ción de un fondo para la investigación en salud intercultural, que elabore pautas específicas para la reflexión, discusión, investigación y docencia en este campo.

La bioética narrativa podría ayudar a entender que para la sabiduría mapuche la dimensión territorial, el respeto a la biodiversidad y el entorno natural (itrofill mongen) es inseparable del buen vivir (küme mongen) individual. Se esta forma la salud sólo se puede alcanzar mediante procesos sociales y relacionales que suponen la armonización de las formas de vida, incrustándolas en normas y criterios basados en relatos, interpretaciones y cosmovisiones compartidas.

\section{REFERENCIAS}

-Ackerknecht, E. H. (1985). Medicina y antropología social: estudios varios, seleccionados por H.H. Walser y H.M. Koelbing, Torrejón de Ardoz: Akal.

-Aguirre Beltrán, G. (1994). Programas de salud en la situación intercultural [1955], Obra Antropológica V, México: FCE / Universidad Veracruzana / Instituto Nacional Indigenista / Gobierno del Estado de Veracruz.

-Álvarez Díaz, A. (2007). Práctica complementaria en salud y recuperación del conocimiento tradicional mapuche. Anales de Antropología, 41 (1), 143-172.

-Arendt, H. (1993). La condición bumana, Barcelona: Paidós.

-Arras, J. D. (1997). Nice Story, But So What? En H. Lindemann Nelson, Stories and their Limits: Narrative Approaches to Bioethics (pp. 65-90). New York: Routledge.

-Beaucham T. L. \& Childress, J. F. (1994). Principles of Biomedical Ethics. (Fourth Edition). New York/Oxford: Oxford University Press.

-Bentele K. (2007). Ethische Aspekte der Regenerativen Medizin. Das Beispiel Morbus Parkinson. Berlin: LIT Verlag.

-Bruner, J. (1991). The Narrative Construction of Reality. Critical Inquiry (18), 1 21.

-Buxó Rey, M. J. (2004). Bioética intercultural para la salud global. Revista de Bioética y Derecho (1), 12-15.

-Callahan, D. (2003). Principalism and Communitarianism. Journal of Medical Etbics, 29 (5), 287-291

-Campos Navarro, R. (2004). Experiencias sobre Salud intercultural. En G. Fernández Juárez (coord.), Salud e interculturalidad en América Latina Perspectivas antropologicas (pp. 129-151). Quito: Abya-Yala.

-Citarella, L. (Comp.) et al. (1995). Medicinas y culturas en La Araucanía, Santiago: Sudamericana.

-Charon, R., (1993). The Narrative Road to Empathy. En H. Spiro et al (eds.), Emphaty and the practice of medicine: beyond pills and the scalpel, New Haven: Yale University Press.

-Charon, R. (1994). Narratives Contributions to Medical Ethics: Recognition, Formulation, Interpretation, and Validation in the Practice of Ethicist. En 
E. R. DuBose, R. P. Hamel and L. J. O'Connell (eds.), A Matter of Principles?, Ferment in U.S. Bioethics (pp. 260-283). Valley Forge: Trinity Press International.

-Charon, R. (2001). Narrative Medicine: Form, Function and Ethics. Annals of internal Medicine, (134), 83-87.

-Charon, R. (2004). Narrative and Medicine. New England Journal of Medicine, 350, (9), 862-864.

-Clouser D. \& Gert, B. (1990). A Critique of Principlism. The Journal of Medicine and Philosophy, (15), 219-236.

-Conill, J. (2006). Ética Hermenéutica. Madrid: Tecnos.

-Cortina, A. (1994). Ética del discurso y bioética. En D. Blanco Fernández, L. Sáez Rueda, J. A. Pérez Tapias (coords.), Discurso y realidad: en debate con K.O. Apel (pp. 75-89). Madrid: Trotta, pp. 75-89.

-Cortina, A. (2007). Ética de la razón cordial. Educar en la ciudadanía en el siglo XXI. Oviedo: Ediciones Nobel.

-Curivil, R. (2008). La Fuerza de la Religión de la tierra. Santiago: Ed. UCSH.

-Cuyul, A. (2013). Salud intercultural y la patrimonialización de la Salud Mapuche en Chile. Morelos: En El Volcán, (22), 7-24.

-Denoux, P. (1994). Pour une nouvelle définition de l'interculturation. En Perspectives de l'inter-culturel (pp. 67-81). París: L'Harmattan/ENS Fontenay-St. Cloud.

-De Rojas, F. (2006). La Celestina, Comedia o tragicomedia de Calisto y Melibea. Castalia, Madrid.

-Dion Stout, M. \& Coloma, C. (1993). Los Pueblos Indigenas y la salud. Documento base. Washington, D.C: Canadian Society for International Health/ OPS- Organización Panamericana de la Salud.

-Domingo Moratalla, A. (2001). El arte de poder no tener razón. La hermenéutica dialógica de Gadamer. Salamanca: UPSA.

-Domingo Moratalla, T. \& Feito Grande, L. (2013). Bioética Narrativa. Madrid: Escolar y Mayo.

-Durkheim, E. (1992). Formas elementales de la vida religiosa. Madrid: Akal.

-Elliot, C. (1998). On Being Unprincipled. Theoretical Medicine and Bioethics, 19, (2), 153-159.

-Fernández Juárez, G. (2011). Claroscuros de la salud intercultural en América Latina. En Nuevo Mundo Mundos Nuevos, Débats [En línea], consulta 18 abril 2014. URL: http:// nuevomundo.revues.org/61188; DOI: 10.4000 /nuevomundo.61188.

-Ferry, J. M. (1996). L'Éthique reconstructive. Paris: Éditions du Cerf.

-Fornet Beancourt, R. (2004). Filosofía intercultural. En R. Salas (coord.), Pensamiento crítico latinoamericano, conceptos fundamentales (pp. 339-414). Santiago: Ed. Universidad Católica Silva Henríquez.

-Gadamer, H. G. (2007). Wahrbeit und Methode. Berlin: Akademie.

-Gillon, B. \& Lloyd, A. (eds.) (1994). Principles of Health Care Ethics. Chichester: John Wiley and Sons. 
-Gracia, D. (2002a). De la bioética clínica a la bioética global: treinta años de evolución. Acta bioethica. [online]. 8, (1), 27-39.

-Gracia, D. (2002b). Aportación de la medicina y a la bioética de la ética narrativa y hermenéutica. En VV. AA.: La bioética, diálogo verdadero (pp. 175-202). Madrid: IV Congreso Nacional, Asociación de Bioética Fundamental y Clínica.

-Haker, H. (2006). Narrative Bioethics. Bioethics in cultural contexts, International Library of Ethics, Law and the New Medicine, (28), 353-376.

-Harris, J. (2003). In praise of Unprincipled Ethics. Journal of Medical Ethics, 29, (5), 303-306.

-Hipócrates (2003). Tratados Hipocráticos I. Sobre la docencia. En J. Sánchez Caro y J. Sánchez Caro, El consentimiento informado en psiquiatría. Madrid: Ediciones Díaz de Santos.

-Laín Entralgo, P. (1964). La relación médico-enfermo : Historia y teoría. Madrid: Editorial Revista de Occidente.

-Lechner, N. (2002). Los nuevos perfiles de la política. Un bosquejo. Nueva Sociedad, (180-181), 263-279.

-Levi Strauss, C (1968). La eficacia simbólica. En Antropología estructural (pp. 168-185). Buenos Aires: Eudeba.

-Lévinas, E. (1997). Totalidad e infinito. Salamanca: Sígueme.

-Macintyre, A. (1981). After Virtue: A Study in Moral Theory, Notre Dame: University of Notre Dame Press.

-Málaga, G. (2013). Las esterilizaciones forzadas, los derechos reproductivos y el consentimiento informado. Revista Peruana de Medicina Exploratoria y Salud Publica, 30, (3), 521-30.

-Marías, J. (1984). El sistema de las libertades. Cuenta y Razón, (15), 9-12.

-Marías, J. (2000). Tratado sobre la convivencia: concordia sin acuerdo. Barcelona: Martínez Roca.

-Marileo, A. (1995). Autoridades Tradicionales y Sabiduría Mapuche. En ¿Modernización o Sabiduria en Tierra Mapuche? (pp. 11-30). Santiago: San Pablo.

-Marileo, A. \& Salas, R. (2011). Filosofía Occidental y Filosofía Mapuche: Iniciando un Diálogo. ISEES, (9), 119-138.

-Menéndez, E. (2009). Esterilizaciones y sectores sociales subalternos en América Latina. El caso mexicano. En G. Fernández Juárez, Salud e Interculturalidad en América Latina. Prácticas quirúrgicas y pueblos originarios (pp. 119-134). Quito: Abya Yala.

-MIDEPLAN (2010). Sistematización de las experiencias de salud intercultural. Santiago: Programa Orígenes.

-Montello, M. (1995). Narrative competence. En H. Lindemann Nelson (ed.), Stories and Their Limits: Narrative Approaches to Bioethic (Reflective Bioethics), Londres: Routledde.

-Montello, M. (2004). Realidad Mental y mundos posibles. Los actos de la imaginación que dan sentido a la experiencia. Barcelona: Gedisa.

-Montgomery, K. (1991). Doctor's Stories: The Narrative Structure of Medical Knowlge, Princeton: Princeton University Press. 
-Montgomery, K. (2006). How Doctors Think: Clinical Judgment and the Practice of Medicine, Nueva York: Oxford University Press.

-Nussbaum, M. (1995). Poetic Justice: the literary imagination and public life. Boston: Beacon Press.

-Nussbaum, M. (2013). Political Emotions: Why Love Matters for Justice. Cambridge, Massachusetts: The Belknap Press of Harvard University Press.

-OPS Organización Panamericana de la Salud (1996). Memoria primer encuentro nacional salud y pueblos indígenas: hacia una politica nacional intercultural en salud, Saavedra - Región de La Araucania. Santiago: Organización Panamericana de la Salud/Organización Mundial de la Salud.

-OPS Organización Panamericana de la Salud (2008). Una visión de salud intercultural para los pueblos indigenas de las Américas. Washington, D.C: Organización Panamericana de la Salud.

-Ortega y Gasset, J. (1975). La historia como sistema. Madrid: Revista de Occidente.

-Pérez Oliva, M (1989). La silla del doctor Marañón. El País, Madrid, 28 de octubre de 1989, p. 23.

-Plessner, H. (1928). Die Stufen des Organischen und der Mensch. Einleitung in die Philosophische Anthropologie, de Gruyter.

-Ricoeur, P. (1996). Sí mismo como otro, Madrid: Siglo XXI.

-Rubinstein, R. A. (1996). Judging the Other. Responding to Traditional Female Genital Surgeries. Hastings Center Report, (26), 31-40

-Salaverry, O. (2010). Interculturalidad en salud. Revista Peruana de Medicina Experimentaly Salud Pública, 27, (1), 80-93.

-Salas, R. (1996). Lo Sagrado y lo bumano. Santiago: San Pablo.

-Salas, R. (2003). Ética Intercultural. Santiago: Ediciones UCSH.

-Schapp, W. (2012). In Geschichten verstrickt. Zum Sein von Mensch und Ding. Fráncfort: Klostermann.

-Siurana, J. C. (2010). Los principios de la bioética y el surgimiento de una bioética intercultural. Veritas, (22), 121-157.

-Sodepaz-Pachakuti (2004). La sabiduría mapuche [En línea], consulta 10 enero 2016, en Rebelión.org, 20 de febrero del 2004.

-Tealdi, J. C. (coord.) (2008). Diccionario Latinoamericano de Bioética. UNESCO, Bogotá: Red Latinoamericana y del Caribe de Bioética - Universidad Nacional de Colombia.

-Toulmin, S. (1981). The Tyranny of Principles. Hastings Center Report, (11), 3139.

-Williams, B. (1987). Ethics and the Limits of Philosophy. London: Fontana.

-Zubiri, X. (1966). Notas sobre la inteligencia humana. Asclepio, Arcbivo Iberoamericano de Historia de la Medicina y Antropología Médica, (18-19), 341-353. 
Sumario: Introducción; 1. Situación de la bioética narrativa; 2. Desde las claves de la medicina narrativa; 3. La razón narrativa y la tradición racio-vitalista; 4. Aportes de la antropología médica y los enfoques interculturales en bioética; 5 . Salud intercultural y complementariedad de sistemas médicos; 6. Dilemas bioéticos en el sistema salud intercultural chileno; 7. La sabiduría ancestral mapuche y sus formas narrativas; Conclusiones; Referencias. 